\title{
lon Chromatography
}




\section{MODERN ANALYTICAL CHEMISTRY}

Series Editor: David Hercules

University of Pittsburgh

ANALYTICAL ATOMIC SPECTROSCOPY

William G. Schrenk

APPLIED ATOMIC SPECTROSCOPY

Volumes 1 and 2

Edited by E. L. Grove

CHEMICAL DERIVATIZATION IN ANALYTICAL CHEMISTRY

Edited by R. W. Frei and J. F. Lawrence

Volume 1: Chromatography

Volume 2: Separation and Continuous Flow Techniques

COMPUTER-ENHANCED ANALYTICAL SPECTROSCOPY

Volume 1: Edited by Henk L. C. Meuzelaar and Thomas L. Isenhour

Volume 2: Edited by Henk L. C. Meuzelaar

ION CHROMATOGRAPHY

Hamish Small

ION-SELECTIVE ELECTRODES IN ANALYTICAL CHEMISTRY

Volumes 1 and 2

Edited by Henry Freiser

LIQUID CHROMATOGRAPHY/MASS SPECTROMETRY

Techniques and Applications

Alfred L. Yergey, Charles G. Edmonds, Ivor A. S. Lewis, and Marvin L. Vestal

MODERN FLUORESCENCE SPECTROSCOPY

Volumes 1-4

Edited by E. L. Wehry

PHOTOELECTRON AND AUGER SPECTROSCOPY

Thomas A. Carlson

PRINCIPLES OF CHEMICAL SENSORS

Jiři Janata

TRANSFORM TECHNIQUES IN CHEMISTRY

Edited by Peter R. Griffiths

A Continuation Order Plan is available for this series. A continuation order will bring delivery of each new volume immediately upon publication. Volumes are billed only upon actual shipment. For further information please contact the publisher. 


\section{lon \\ Chromatography}

\section{Hamish Small}

Formerly Research Scientist

The Dow Chemical Company

Midland, Michigan

Springer Science+Business Media, LLC 


\section{Library of Congress Cataloging in Publication Data}

Small, Hamish.

Ion chromatography / Hamish Small.

p. $\quad \mathrm{cm}-$ (Modern analytical chemistry)

Includes bibliographical references.

ISBN 978-1-4899-2544-2

ISBN 978-1-4899-2542-8 (eBook)

DOI 10.1007/978-1-4899-2542-8

1. Ion exchange chromatography. I. Title. II. Series.

QD79.C453S63 1990

89-39790

$543^{\prime} .0893-\mathrm{dc} 20$

CIP

() 1989 Springer Science+Business Media New York

Originally published by Plenum Press, New York in 1989.

Softcover reprint of the hardcover 1st edition 1989

All rights reserved

No part of this book may be reproduced, stored in a retrieval system, or transmitted in any form or by any means, electronic, mechanical, photocopying, microfilming, recording, or otherwise, without written permission from the Publisher 
To Beryl, Deborah, and Claire 


\section{Preface}

Bewitched is an odd word with which to begin a chemical textbook. Yet that is a fair description of how I reacted on first learning of ion exchange and imagining what might be done with it. That initial fascination has not left me these many years later, and it has provided much of the motivation for writing this book. The perceived need for a text on the fundamentals of ion chromatography provided the rest.

Many readers will have a general idea of what ion chromatography is and what it does. Briefly, for those who do not, it is an umbrella term for a variety of chromatographic methods for the rapid and sensitive analysis of mixtures of ionic species. It has become highly developed in the last decade, and while it is now routinely used for the determination of organic as well as inorganic ions, its initial impact was greatest in the area of inorganic analysis. In the past the determination of inorganic ions, particularly anions, meant laborious, time-consuming, and often not very sensitive "wet chemical" methods. In the last ten years that has changed radically as ion chromatography has supplanted these older methods.

While ion chromatography (IC) is relatively new, the principles that underlie its practice and guide the development of its several parts have been laid down over decades of research in many diverse areas of chemistry. A primary purpose of this book is to provide a systematic account of this fundamental chemistry and the principles that underlie it.

Ion chromatography is a practical art, and this book is about practical matters. So although there is an emphasis on basic principles, I have placed equal importance on showing the direct relationship of the underlying scientific concepts to everyday practical matters of IC, such as the design and synthesis of ion exchange resins and the solution of real chromatographic problems. I have treated detection in IC at some length, in the first place because detection stands equal in importance to separation in modern chromatography, and second because new methods of detection are largely responsible for the renaissance of ion exchange in analytical chemistry. The book has also given me the opportunity to address 
the specific area of conductometric detection, which, in IC, is still a source of some misunderstanding.

To whom is the book addressed and to whom might it appeal? Modern instrumentation, with its emphasis on operator convenience and its ability to automate, has been a tremendous boon to the analyst in increased productivity and decreased tedium. On the other hand, it tends increasingly to insulate the user from the underlying science, so texts and the like that emphasize the theoretical bases of methods provide a necessary counterbalance to this "black box" tendency. I believe such texts to be especially necessary in chromatography, where the operator is such an active participant in the analytical process, in that decisions must often be made on such matters as separation method, stationary and mobile phases, and modes of detection. So in considering to whom the book should appeal I always had in mind the practicing chromatographer. I hope also that the book will be of help to those who are involved in the design of new chromatographic phases since I have endeavored to treat the synthesis of exchangers and the origins of ion selectivity at some length. And an early chapter on the chromatographic process is intended as a bridge that practitioners of LC and GC will find useful in making the transition to ion exchange chromatography.

In the research phase of Ion Chromatography, and particularly in the writing of the book, I have been keenly aware of the rich hinterland from which we drew much of our resources. I hope the book goes at least a short way in recognizing those who, though they could not know it at the time, have contributed so significantly to the birth and success of this most recent offspring of Ion Exchange.

ACKNOWLEDGments. I am especially indebted to Dr. Ed. Johnson and Ted Miller for their critical reading of the entire manuscript and for the many improvements that they suggested. Also my sincere thanks go to Rosanne Slingsby and Nancy Jensen for their help with many of the figures. 


\section{Contents}

Chapter 1

Introduction

1.1. Background to Ion Chromatography $\ldots \ldots \ldots \ldots \ldots \ldots \ldots \ldots \ldots \ldots \ldots \ldots \ldots \ldots \ldots \ldots \ldots$

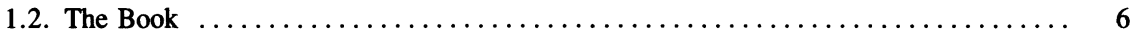

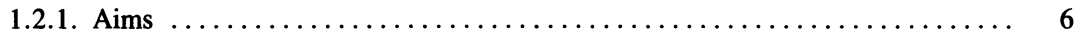

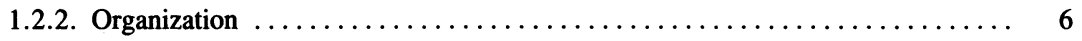

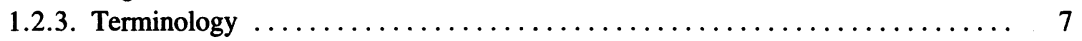

References $\ldots \ldots \ldots \ldots \ldots \ldots \ldots \ldots \ldots \ldots \ldots \ldots \ldots \ldots \ldots \ldots \ldots, 8$

Chapter 2

\section{The Chromatographic Process}

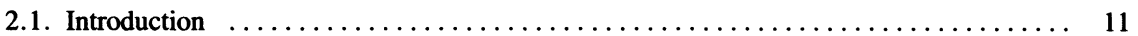

2.2. A Description of a Chromatographic Separation $\ldots \ldots \ldots \ldots \ldots \ldots \ldots \ldots \ldots \ldots \ldots \ldots$

2.3. Thermodynamic Aspects of Chromatographic Separation $\ldots \ldots \ldots \ldots \ldots \ldots \ldots \ldots, 13$

2.3.1. Distribution of a Solute between Two Phases $\ldots \ldots \ldots \ldots \ldots \ldots \ldots \ldots \ldots$

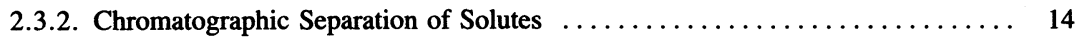

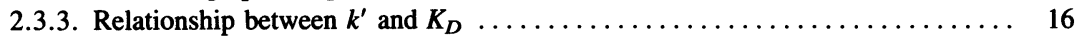

2.3.4. Variation of $K_{D}$ with Concentration-Nonideal Behavior $\ldots \ldots \ldots \ldots \ldots \ldots, 17$

2.3.5. Isotherms and Peak Shape in Chromatography $\ldots \ldots \ldots \ldots \ldots \ldots \ldots . \ldots . \ldots . \ldots 20$

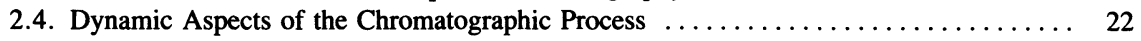

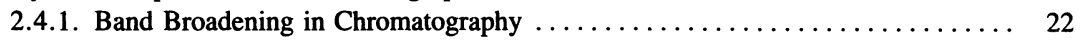

2.4.2. Band Broadening_-Some Experimental Observations $\ldots \ldots \ldots \ldots \ldots \ldots \ldots 23$

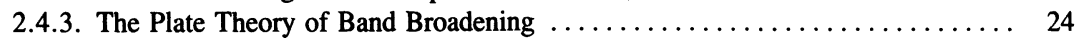

2.5. Rate Theories of Band Broadening $\ldots \ldots \ldots \ldots \ldots \ldots \ldots \ldots \ldots \ldots \ldots \ldots \ldots \ldots \ldots \ldots \ldots, 31$

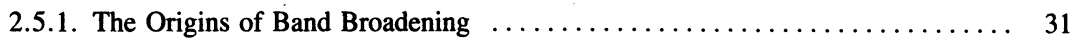

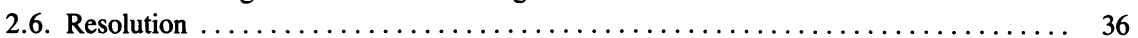

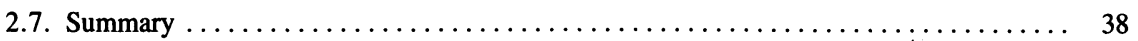

References $\ldots \ldots \ldots \ldots \ldots \ldots \ldots \ldots \ldots \ldots \ldots \ldots \ldots \ldots \ldots \ldots \ldots \ldots \ldots \ldots \ldots \ldots, 38$

Chapter 3

The Materials of Ion Chromatography

3.1. Introduction 


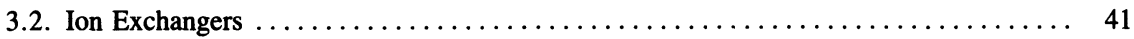

3.2.1. Polymer Precursors of Ion Exchange Resins $\ldots \ldots \ldots \ldots \ldots \ldots \ldots \ldots \ldots \ldots$

3.2.2. Cation Exchange Resins $\ldots \ldots \ldots \ldots \ldots \ldots \ldots \ldots \ldots \ldots \ldots \ldots \ldots \ldots \ldots \ldots$

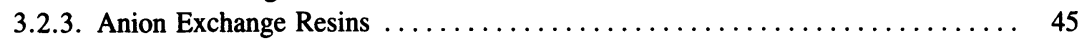

3.2.4. Surface Agglomeration Method for Preparing Low-Capacity Ion Exchangers . . 46

3.2.5. Silica- and Methacrylate-Based Ion Exchangers $\ldots \ldots \ldots \ldots \ldots \ldots \ldots \ldots \ldots$

3.2.6. Exchangers with Weakly Functional Groups $\ldots \ldots \ldots \ldots \ldots \ldots \ldots \ldots \ldots \ldots$

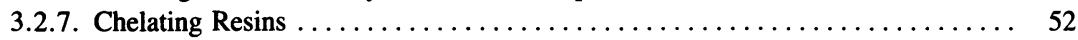

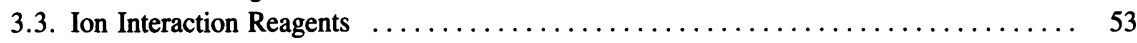

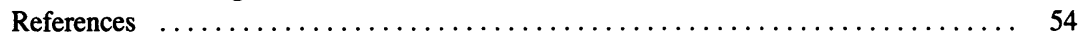

Chapter 4

\section{Ion Exchange in Ion Chromatography}

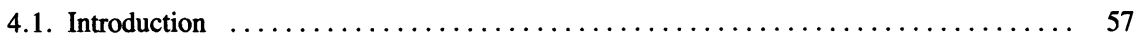

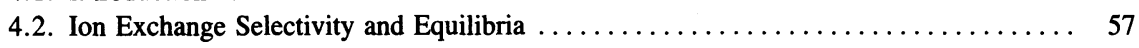

4.2.1. Selectivity and the Selectivity Coefficient $\ldots \ldots \ldots \ldots \ldots \ldots \ldots \ldots \ldots$

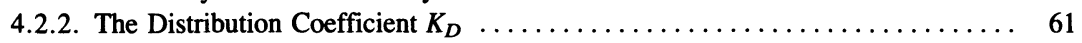

4.2.3. The Donnan Potential and Ion Exchange Equilibrium $\ldots \ldots \ldots \ldots \ldots \ldots$

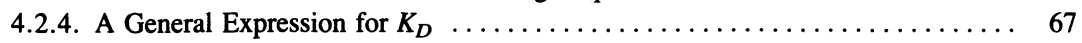

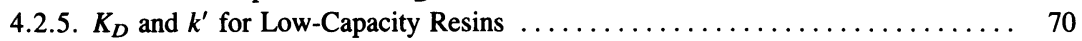

4.2.6. Effects Attributable to the $\mathrm{pH}$ of the External Phase $\ldots \ldots \ldots \ldots \ldots \ldots \ldots$

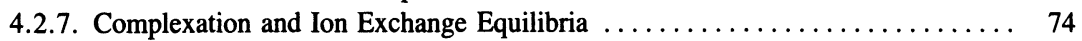

4.2.8. Theories on the Causes of Ion Exchange Selectivity $\ldots \ldots \ldots \ldots \ldots \ldots \ldots$

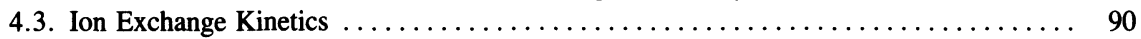

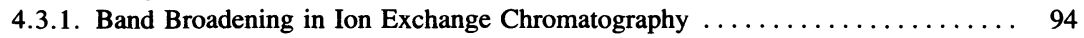

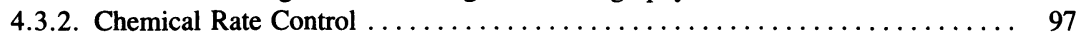

4.4. Ion Exchange Chromatography $\ldots \ldots \ldots \ldots \ldots \ldots \ldots \ldots \ldots \ldots \ldots \ldots \ldots \ldots \ldots$

4.4.1. Elution Development $\ldots \ldots \ldots \ldots \ldots \ldots \ldots \ldots \ldots \ldots \ldots \ldots \ldots \ldots \ldots \ldots \ldots$

4.4.2. Frontal Analysis $\ldots \ldots \ldots \ldots \ldots \ldots \ldots \ldots \ldots \ldots \ldots \ldots \ldots \ldots \ldots \ldots \ldots$

4.4.3. Displacement Development $\ldots \ldots \ldots \ldots \ldots \ldots \ldots \ldots \ldots \ldots \ldots \ldots \ldots \ldots \ldots . \ldots 9$

4.4.4. Peak Shape in Elution Development $\ldots \ldots \ldots \ldots \ldots \ldots \ldots \ldots \ldots \ldots \ldots \ldots$

4.4.5. Base-Line Disturbances, "System"' Peaks . . . . . . . . . . . . . . . . 104

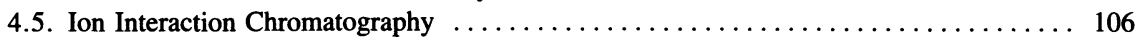

4.5.1. The Electrical Double Layer $\ldots \ldots \ldots \ldots \ldots \ldots \ldots \ldots \ldots \ldots \ldots$

4.5.2. The "Capacity" of the Adsorbed Double Layer $\ldots \ldots \ldots \ldots \ldots \ldots \ldots \ldots \ldots$

4.5.3. Retention of Surface Active Eluites $\ldots \ldots \ldots \ldots \ldots \ldots \ldots \ldots \ldots \ldots \ldots \ldots$

4.5.4. IIC and IEC $-\mathrm{A}$ Comparison $\ldots \ldots \ldots \ldots \ldots \ldots \ldots \ldots \ldots \ldots \ldots \ldots \ldots$

References $\ldots \ldots \ldots \ldots \ldots \ldots \ldots \ldots \ldots \ldots \ldots \ldots \ldots \ldots \ldots \ldots \ldots \ldots$

\section{Chapter 5}

\section{Ion Exchange Resins in Liquid Partition Chromatography}

5.1. Introduction $\ldots \ldots \ldots \ldots \ldots \ldots \ldots \ldots \ldots \ldots \ldots \ldots \ldots \ldots \ldots \ldots \ldots$

5.2. Sorption Equilibria on Ion Exchange Resins $\ldots \ldots \ldots \ldots \ldots \ldots \ldots \ldots \ldots \ldots \ldots \ldots$

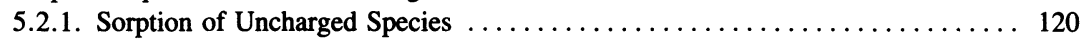

5.2.2. Sorption of Strong Electrolytes $\ldots \ldots \ldots \ldots \ldots \ldots \ldots \ldots \ldots \ldots \ldots \ldots \ldots$

5.2.3. Thermodynamic Treatment of Electrolyte Distribution and Ion Exclusion . . . . 125

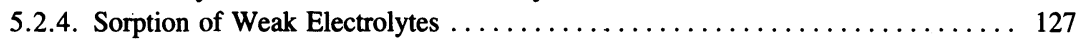




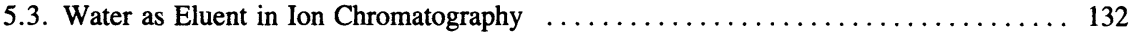

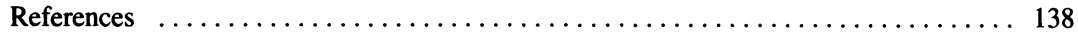

Chapter 6

\section{Detection-General}

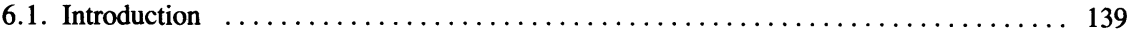

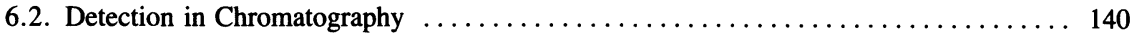

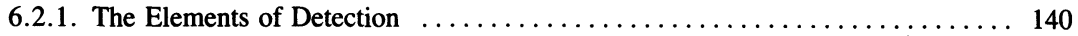

6.2.2. The Requirements of a Detector .......................... 141

6.2.3. Sensitivity, Noise, and Limits of Detectability $\ldots \ldots \ldots \ldots \ldots \ldots \ldots \ldots 142$

6.2.4. Mobile Phase as a Source of Noise and Limitations on Detectability ....... 144

6.2.5. Definition of Detectability ............................. 147

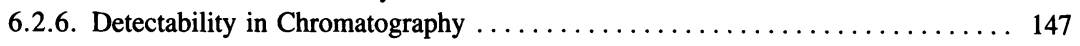

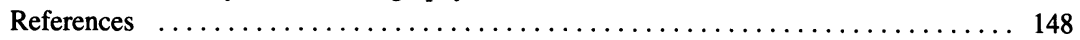

Chapter 7.

\section{Conductometric Detection}

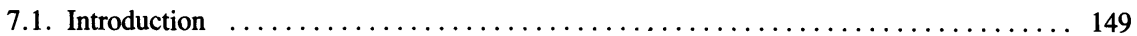

7.2. Electrolyte Conductance-Theory and Measurement $\ldots \ldots \ldots \ldots \ldots \ldots \ldots \ldots \ldots 151$

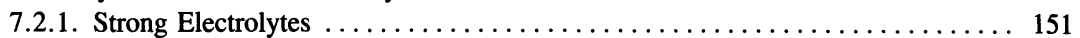

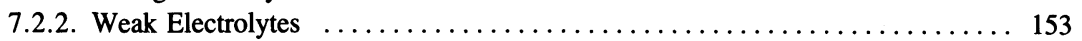

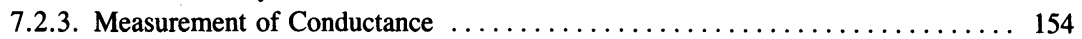

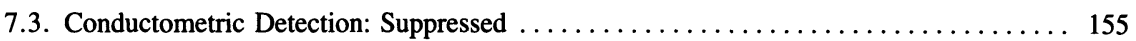

7.3.1. Eluent Suppression .................................. 155

7.3.2. Exhaustion and Regeneration of Column Suppressors ............... 158

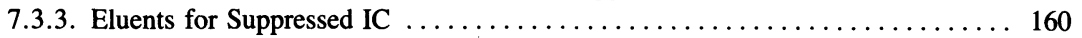

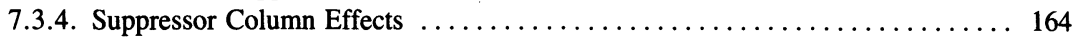

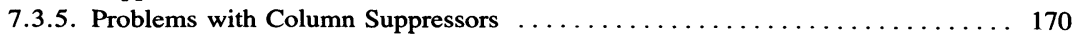

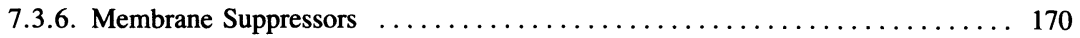

7.3.7. Removal of $\mathrm{CO}_{2}$ from Column Effluent .................... 174

7.3.8. Nonlinearity Effects in Suppressed Conductometric Systems ............ 175

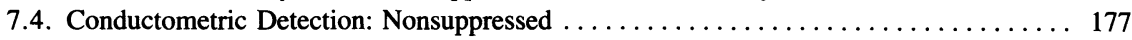

7.4.1. The Basis of Nonsuppressed Conductometric Detection .............. 178

7.4.2. Eluent Choice .................................. 179

7.5. A Comparison of Suppressed and Nonsuppressed Conductometric Detection ........ 180

7.5.1. Simulated Experiments Using Suppressed and Nonsuppressed Methods ...... 181

7.6. Gradient Elution and Conductometric Detection ........................ 186

7.7. Conductometric Detection in Ion Chromatography - A Summary . . . . . . . . . . . 187

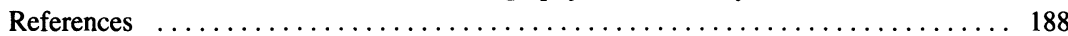

\section{Chapter 8}

\section{Other Modes of Detection}

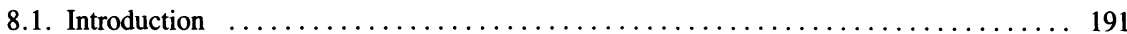

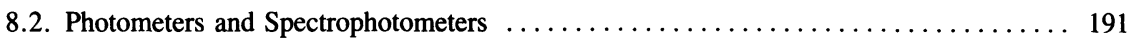

8.2.1. Principles of Photometric Detection . . . . . . . . . . . . . . . . . 191 
8.2.2. Sensitivity of Spectrophotometers $\ldots \ldots \ldots \ldots \ldots \ldots \ldots \ldots \ldots \ldots \ldots \ldots$

8.2.3. Photometric Detection with Postseparation Derivatization . . . . . . . . . 195

8.2.4. Indirect Photometric Detection (IPD) $\ldots \ldots \ldots \ldots \ldots \ldots \ldots \ldots \ldots \ldots \ldots \ldots$

8.3. Electrochemical Detection $\ldots \ldots \ldots \ldots \ldots \ldots \ldots \ldots \ldots \ldots \ldots \ldots \ldots \ldots \ldots \ldots \ldots \ldots$

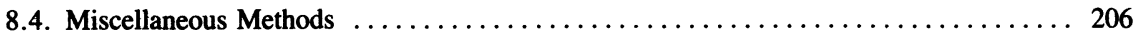

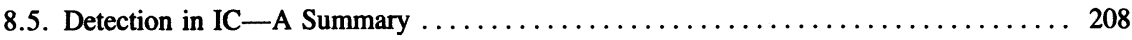

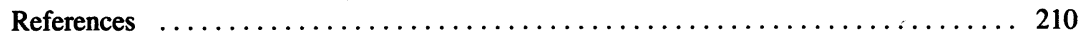

Chapter 9

Selected Applications of Ion Chromatography

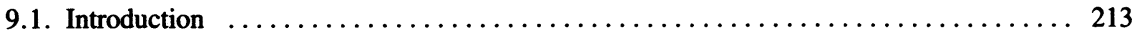

9.2. Conductometric Detection in the IC of Common Inorganic Anions $\ldots \ldots \ldots \ldots \ldots 213$

9.3. Analysis of Alkali Metal and Alkaline Earth Metal Ion Mixtures $\ldots \ldots \ldots \ldots \ldots .218$

9.3.1. Alkali Metal and Alkaline Earth Metal Ions Together ............. 218

9.3.2. A "Mechanical" Solution .............................. 221

9.3.3. Alkali Metal or Alkaline Earth Metal Ions Separately ............. 223

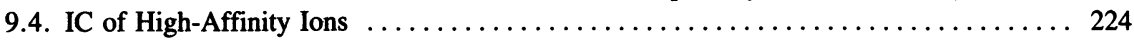

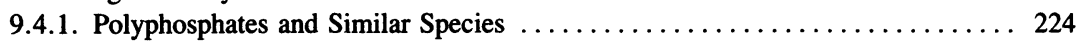

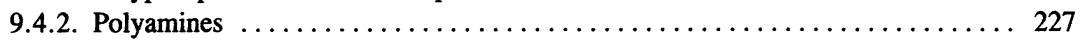

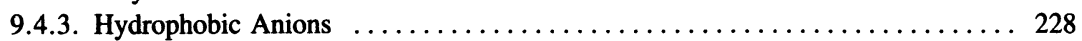

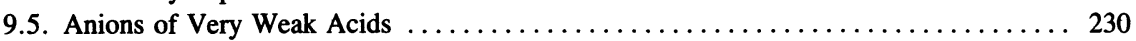

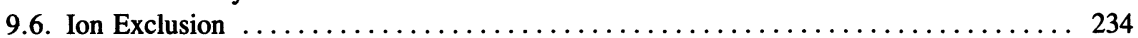

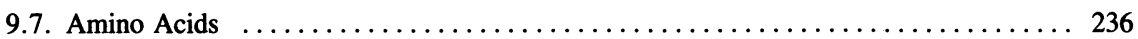

9.7.1. Acid-Base Chemistry of Alpha-Amino Acids $\ldots \ldots \ldots \ldots \ldots \ldots \ldots \ldots \ldots 236$

9.7.2. Ion Exchange Separation of Amino Acids ................... 239

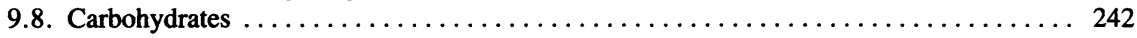

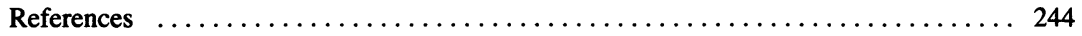

\section{APPENDIXES}

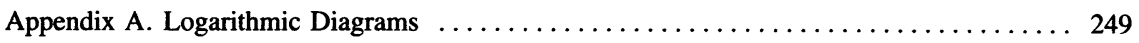

Appendix B. Limiting Equivalent Conductivities of Ions at $25^{\circ} \mathrm{C} \ldots \ldots \ldots \ldots \ldots \ldots . \ldots 259$

Appendix C. Conductance of Carbonic Acid as it is Neutralized by Sodium Hydroxide . . . 261

Appendix D. Conductance of a Strong Acid in a Background of Weak Acid .......... 263

Appendix E. Ionization Constants and $\mathrm{pH}$ Values at the Isoelectric Points (pI) of the

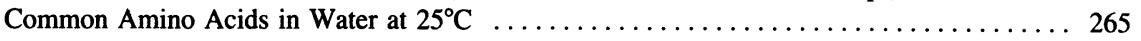

Appendix F. The Structure of Common Alpha-Amino Acids . . . . . . . . . . . . . . . . 267

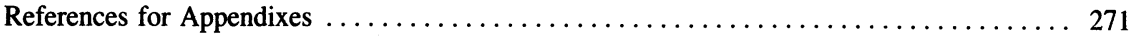

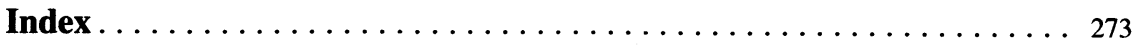

